\title{
TCP Congestion Control in Wired cum Wireless Networks
}

Neeraj Sharma

\begin{abstract}
Since the introduction of "TCP for congestion control in computer networks", this concept has been widely used in wired networks. TCP congestion control has its different variants in various types of networks. Many other protocols are also introduced, which are derived from "TCP Congestion Control" concept for wired networks. TCP congestion control is successfully implemented in wired networks and is sill improving but it's not the case with wireless networks because of compatibility and non-functional issues. During last two decades students, researchers and scholars are continuously embracing and improving TCP congestion functionality both in wired and wireless networks by focusing on four modules of congestion control algorithms i.e. slow start, congestion avoidance, and fast recovery and fast retransmit. Proposal is to build a system that identifies the network type and behave accordingly
\end{abstract}

\section{General Terms}

TCP, Congestion Control, Wireless networks, Wired Networks, maintaining congestion window size, slow start, congestion avoidance, and fast recovery, fast retransmit.

\section{INTRODUCTION}

TCP or Transmission Control Protocol is one of the prevailing techniques used in computer networks for the reliable transmission of data or packets or segments over networks. TCP generally complements Internet Protocol. This protocol is generally used and implemented by many internet applications such as file transfer, World Wide Web, electronic mailing, etc. Data transfer in networks using TCP is very complicated task. Congestion Control is an approved mechanism that detects an optimum bandwidth for transmitting data segments over networks. Many researchers have been made till date in the direction of improving TCP performance in computer networks.

\section{CONGESTION CONTROL ALGORITHMS}

TCP Congestion Control Algorithms are basically grouped into four categories, namely, SLOW START, CONGESTION AVOIDANCE, FAST RETRANSMIT and FASTRECOVERY [2].

TCP was introduced to wired networks because of the reliability of network type. Loss in wired networks was solely because of congestion but it is not the case with wireless networks. There may be number of other reasons including congestion for the packet loss in wireless networks e.g. node failure, BER, mobility, etc. Leaving this discussion for further sections let us get briefly introduced to congestion control algorithms:

\subsection{Slow Start}

Slow Start is mechanism used for TCP implementation to control the flow of data stream over networks from the sender's end. It is also sometimes known as sender based flow control. Rate at which sender can transmit data over network depend upon the rate of the acknowledgments issued by the receiver. On the network establishment the Congestion Window on sender side is set to unity which is the MSS (maximum segment size). On receiving an acknowledgment from the receiver the congestion window is set to double of its size. Size of the congestion window is increase until it attains a particular threshold value or the network enters congestion state whichever comes first [11]. At some point of time network may change state and packet may start dropping. At this point of time sender will go into Congestion Avoidance phase.

\subsection{Congestion Avoidence}

During the initiation of data transfer over a networks slow start is used, however network may reach a point where the packets may start dropping. In this case Congestion Avoidance is used to slow down the data transfer. Congestion Avoidance is practically used in conjunction with Slow Start. In Congestion Avoidance algorithm a duplicate acknowledgement signals the sender about the occurrence of congestion. Sender then immediately sets the congestion window to half of its size. Size of congestion window is increased linearly. This mechanism will allow the sender to grow its transmission in more gradual manner as it reaches the point where congestion previously occurred.

\subsection{Fast Retransmit}

When duplicate acknowledgements are received at the senders end it is not clear that whether the duplicity is because of packet failure or it just arrived out of order. However no more than two duplicate acknowledgements should arrive if the packets were out of order. If three duplicate acknowledgments arrive at sender, it does not wait for timer to expire and immediately retransmits the missed packet. This is known a FAST RETRANSMIT.

\subsection{Fast Recovery}

Receiving duplicate acknowledgement does not mean that there is some serious network congestion because having duplicate acknowledgement implies that data is still flowing in network. Therefore instead of going all the way back to slow start phase sender resumes transmission with a larger window and start increasing it gradually as in the case of congestion avoidance. This will result in increased throughput. This phenomenon is referred to as FAST RECOVERY. 


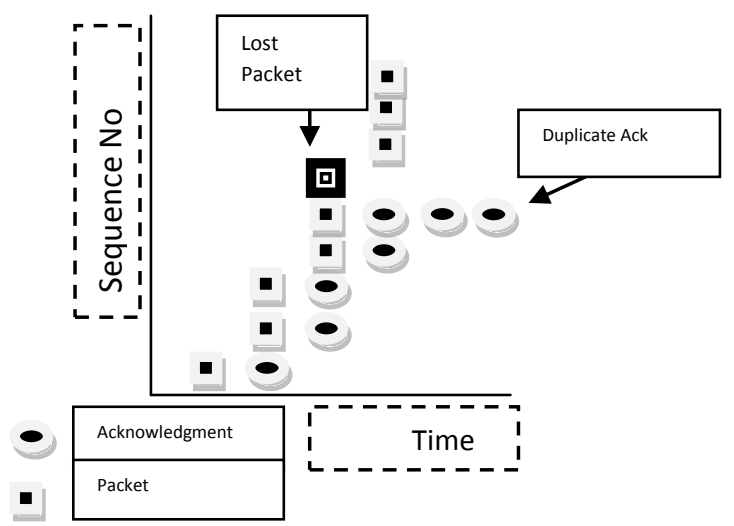

Fig 1: Duplicate Acknowledgment

\section{TCP CONGESTION CONTROL IN WIRELESS NETWORKS}

TCP was initially introduced to wired computer networks and was successfully implemented because of the advantage of reliability of wired networks. But it is not the case with wireless networks because of some non functional issues. Following are some major reasons why TCP implementation is cumbersome task in wireless networks:

- Bandwidth: The total link capacity is shared by all nodes which can work within transmission range, including hidden nodes. Since collisions drastically decrease the throughput, it is desired to have knowledge of the total available bandwidth. Thus, various predictive algorithms have been proposed for that. These limitations are identified as the Throughput Upper Limit (TUL) and the Delay Lower Limit (DLL).

- Latency: Latency in a wireless medium is greater than in a wired one. The factors that influence latency are overhead added by both physical layer and link layer protocols, propagation delay and the retransmission policy implemented at the link layer.

- Channel losses: Wireless channels suffer from fading caused by interference with other channels. There is significant amount of difference in the BER (Bit Error Rate) values of wired and wireless channels. The typical scheme used to recover from losses is the link layer ARQ (Automatic Response reQuest).

- Mobility: The most common network setting is the infrastructured BSS connected to a fixed network via a Base Station (BS). Handoff requires that all the information linked to user activities be transferred from Base Station to the next to prevent the termination of service provided to the mobile user.

- $\quad T C P$ : The problems that arise in the usage of TCP over wireless networks are due to their time-variant characteristics such as fading, shadowing, node mobility, hand-offs, limited available bandwidth and large RTTs, as well as low reliability. TCP performs poorly in such environments since they were originally designed for use on wired networks, which are characterized by stable links in which packet losses are mainly limited to congestion.

\section{ENHANCEMENT OF TCP THROUGHPUT IN WIRED CUM WIRELESS NETWORKS}

There is one property of TCP congestion control algorithm that it uses Packet Loss as to signal network congestion. This property works fine in wired networks but it becomes a contradiction in wireless networks sometimes. Since signal noise, fading ratio channel, BER, node mobility, node failure and many other non functional issues may also be the reasons for packet loss in wireless networks [12]. This creates problems in TCP Reno (Congestion Control Algorithm) [6] since it does not possess the measures to differentiate congestion loss from wireless loss. Three alternative approaches: end-to-end (e-to-e), split connection, and localized link layer methods were carefully contrasted. The split-connection approach violates the semantics of e-to-e reliability. Second, this approach requires a lot of state maintenance at the base station. Westwood TCP (TCPW) is a new congestion control algo that is based on e-to-e bandwidth calculation.

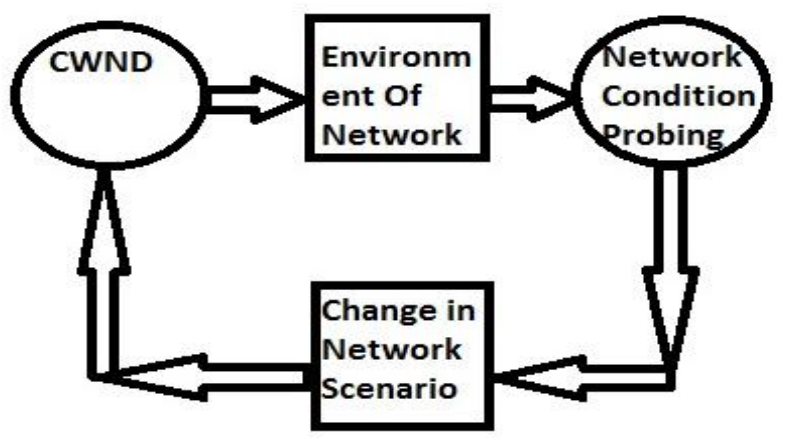

Fig 2: Block Diagram

\section{PROBLEM FORMULATION}

The TCP congestion avoidance algorithm is mainly the basis for congestion control in the Internet. Problem occurs when a number of parallel TCP flows are experiencing port queue buffer tail-drops. Then TCP's automatic congestion avoidance mechanism is not enough [1]. All flows that experience port queue buffer tail-drop will begin a TCP retrain at the same moment - this is known as TCP global synchronization.

The proposed scheme when operating in wireless mode exploits the SNR ratio of the communication line to decide whether a lost packet was due to congestion or error loss. On the establishment of TCP connection, the congestion algorithm starts the congestion window cwnd to one segment. Then, the first bit of the reserved field is set according to the type of the link, i.e., $b=0$ for wired and $b=1$ for wireless connection. Segments are then sent to the receiver. When they are successfully acknowledged, the congestion window cwnd is increased by one segment, making its size two segments, then four segments, then eight segments, and so on doubling each time. When congestion occurs, network will start losing packets, triggering a timeout at the sender. In this situation, the sender instantaneously checks the reserved bit $b$. If it is equal to 0 (wired link), then timeout is considered to be because of congestion. The size of the congestion window (cwnd) would be set to half of the current size and the sender resumes the flow of packets [3]. In contrast, if the reserved bit $b$ is equal to 1 
(wireless link), the SNR(Signal To Noise Ratio) of the connection is checked. In case it is within a high range, i.e., greater than $5 \mathrm{~dB} \quad(\mathrm{SNR}>5 \mathrm{~dB})$, then timeout is considered to be because of congestion and the congestion window size is halved by one segment and next packet is sent. However, if SNR ratio is within a low range, i.e., less than $5 \mathrm{~dB} \quad(\mathrm{SNR}<5 \mathrm{~dB})$, then timeout is considered to be because of error and the timed-out packet is retransmitted to the receiver [5]. The flowchart of the proposed congestion algorithm is depicted in Figure 4; while, its pseudo-code is outlined as follows:

1. Initialize congestion window cwnd to one.

2. Start sending data packets to receiver.

3. For each acknowledgement received, increment congestion window cwnd by value of one segment.

4. Then each packet acknowledged is checked for network type.

1. If it is a wired link, then update cwnd to half of its previous size (indicating congestion)

2. Else if wireless link, then check the reliability of the network.

1. If reliable, then apply Fast Recovery Algorithm (indicating congestion)

2. Else if unreliable, then don't change the current size of cwnd, and retransmit packet (indicating an error)



Fig 2: Congestion Control in Hybrid Systems

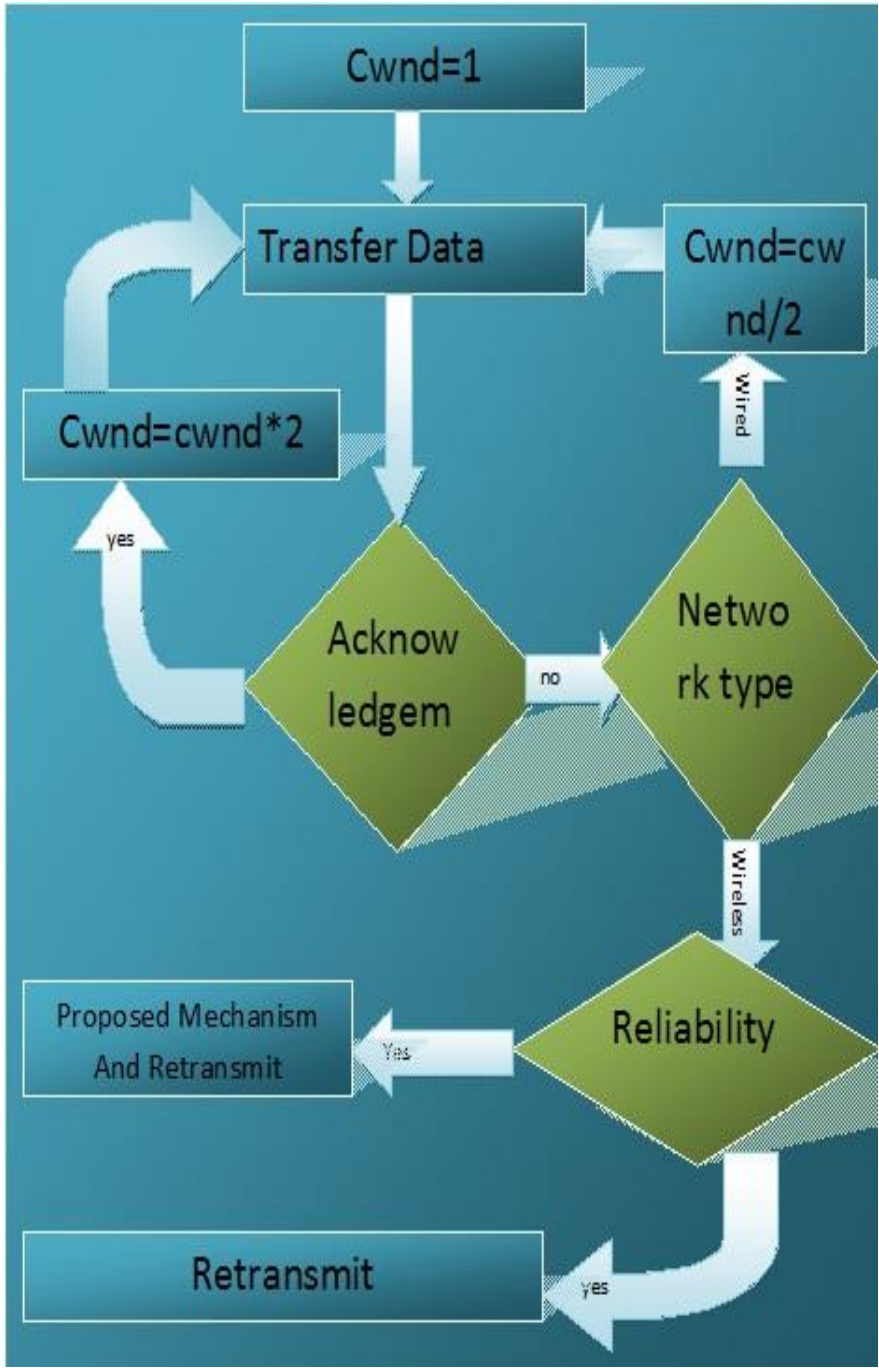

Fig 4: Flowchart Of Proposed System

\section{CONCLUSION}

In ad hoc networks, there exists many external interference which causes multi-path fading and shadow effect because wireless links are open loss mediums, moreover high BER exists in channels arising from selecting open band which causes packet loss and damage in ad hoc networks, accordingly the sender takes those non-congestion cases for network congestion case. Node mobility in ad hoc networks may cause route disconnection and route failure in which route failure induced intermediate node to discard packets. As a result, the TCP sender will take route failure for congestion state and take some unnecessary actions according to congestion control mechanism. In addition, route failure causes the sender to discover new route path using route algorithm at network layer, the time used will be longer than RTO, then the sender enters slow start phase. Proposed approach is worked upon in such a way that it automatically determines the network type and behaves accordingly. Acknowledgement failure in wired networks is mostly due to congestion. But in wireless networks there may be number of reasons like node failure, nod mobility, high BER, etc. So this paper proposes a scheme by which we can improve throughput of wired cum wireless systems. The phenomenon of congestion control is hoped to maintain the size of congestion window in between half of its size and congestion threshold value. 


\section{ACKNOWLEDGMENT}

It gives me immense pleasure to express my deepest sense of gratitude and sincere thanks to my highly respected and esteemed guide Dr. Manish Mann(CSE), LRIET, SOLAN for their valuable guidance, encouragement and help for completing this work. Their useful suggestions for this whole work and co-operative behavior are sincerely acknowledged. I also wish to express my gratitude to Mr. Ravinder Thakur for his kind hearted support. I am also grateful to Dr. Manish Mann, Coordinator of M. Tech. (CSE) for his constant support and guidance. I also wish to express my indebtedness to my parents as well as my family member whose blessings and support always helped me to face the challenges ahead. At the end I would like to express my sincere thanks to all my friends and others who helped me directly or indirectly during this project work.

\section{REFERENCES}

[1] Anup K. Ghosh, Amitava Mukherjee, Debashis Saha (4 September 2008) 'TCP throughput enhancement in wired-cum-wireless network'

[2] Ghassan A. Abed*, Mahamod Ismail, Kasmiran Jumari (27 October 2011) 'Exploration and evaluation of traditional TCP congestion control techniques'

[3] Hualiang Chen*, Zhongxin Liu, Zengqiang Chen, Zhuzhi Yuan(16 October 2006) 'Extending TCP congestion control to multicast in Wireless Sensor Networks'

[4] JANG-PINGSHEU, LI-JENCHANG AND WEI-KAIHU (JOURNAL OF INFORMATION SCIENCE AND ENGINEERING 25, 1103-1119 (2009)) 'Hybrid Congestion Control Protocol'.
[5] Youssef Bessil (June, 2012) 'TCP Congestion Control Scheme for Wireless Networks based on TCP reserved field and SNR ratio'

[6] Saleem-ullah Lar, Xiaofeng Liao (11 October, 2011) 'An initiative for a classified bibliography on TCP/IP congestion control'.

[7] Shao Liu, Tamer Bas sar, R. Srikant(7 December 2007) 'TCP-Illinois: A loss- and delay-based congestion control algorithm for high-speed networks'

[8] Stefan Savage, Neal Cardwell, David Wetherall, and Tom Anderson (October 1999), 'TCP Congestion Control with a Misbehaving Receiver, ACM Computer Communications Review'

[9] V. Jacobson, R. Braden and D. Borman. (May 1992) 'TCP Extensions for High Performance,

RFC 1323 '.

[10] Venkataramana Badarla, C. Siva Ram Murthy (20 January 2011) 'Learning-TCP: A stochastic approach for efficient update in TCP congestion window in ad hoc wireless networks'

[11] Vincent Lucas, Jean-Jacques Pansiot, Dominique Grad, Benoît Hilt (7 November 2012) 'Robust and fair Multicast Congestion Control(M2C)'

[12] Yanping Teng, Haizhen Wang, Mei Jing, Zuozheng Lian(2011) ' A Study of Improved approaches for TCP Congestion Control in Ad Hoc Networks' 